\title{
Application Of Strongly Tracking Kalman Filter In MEMS Gyroscope Bias Compensation
}

\author{
Liu Hong Dan ${ }^{1, a, *}$, Shu Xiong Ying ${ }^{1, b}$ and Li Xi Sheng ${ }^{2, c}$ \\ School of Automation and Electrical Engineering, University of Science and Technology Beijing, \\ Beijing 100083, China \\ a15350615806@163.com, ${ }^{\text {a limosine@sohu.com, }{ }^{c} 1 x s @ u s t b . e d u . c n ~}$ \\ *Corresponding author
}

Keywords: MEMS gyroscope, gyro bias, strong tracking adaptive Kalman filter, error compensation

\begin{abstract}
In this paper, we make a research about a integrated system. The system consists of two main parts: micro-electro-mechanical systems (MEMS) gyroscopes and compass. In order to eliminate the temperature drift bias of MEMS gyroscope, we use a strong tracking Kalman filter and chooses an adaptive algorithm. We established a model about the bias of the temperature of the gyroscope. The parameters of the model are selected for the state variables to change intelligently with the temperature to increase the precision of the MEMS gyroscope ${ }^{[1-2]}$. In the static temperature experiment, the compensated heading error is less than $0.7^{\circ}$. We can draw a conclusion that the traditional Kalman filter compensation method, multiple linear regression compensation method, improved least squares method and strong tracking Kalman adaptive filtering algorithm all can compensate the gyroscope drift bias, but the adaptive filtering algorithm can be more accurate about the compensation of MEMS gyro drift bias, and eliminate the impact of temperature on its accuracy.
\end{abstract}

\section{Introduction}

As one of the most commonly used navigation methods, Inertial navigation has been in a variety of markets such as aerospace and other aspects of applications. As the most important part of the inertial navigation device, MEMS inertial sensors with diminutive size, low cost and low power consumption are more widely used in the middle and low level market. In the integrated navigation systems, they are mainly used to measure the angular velocity of the carrier, and the information obtained from the accelerometer can be used to calculate the course, posture and position of the carrier. The gyroscope is sensitive to temperature variations, so the surrounding temperature variation leads to the bias drift of the gyroscope. In addition the error will accumulate with time. Therefore, it is necessary to improve the compensation of MEMS gyroscope, reduce the influence of error and improve its measurement accuracy.

Since the bias drift is not linear with temperature and MEMS gyroscopes have different production parameters, the result is not accurate with the same parameters to compensate the bias 
drift. At present, Kalman filter and its various extended filtering algorithms are adopted at home and abroad, and the correctness of these methods is verified by experiments. However, due to a variety of methods are used individually, the compensation effect of the bias drift is susceptible to external interference. Thus, we present an adaptive algorithm for a integrated navigation system to compensate the bias drift of MEMS gyroscopes for different temperatures and environments. This paper mainly uses the strongly tracking Kalman filter to complete the adaptive compensation of the gyro bias drift. Firstly, a simplified bias model based on the temperature is built. Then, the strongly tracking Kalman filter is built based on the model. The method is to combine the real-time updating data of the magnetic compass with accelerometer in the integrated navigation system, and establishes a new filtering equation, and selects appropriate state variables and observations. It reduces the workload of calibration and solves the problem that the system parameters can not be updated timely in a short time.

\section{The Model of MEMS Gyroscope Drift}

The analysis shows that the noise of MEMS gyroscope is mainly composed of random noise(high-frequency)and gyroscope $\operatorname{drift}($ low-frequency, slow-changing), so the angular velocity measurement model can be expressed as:

$$
\omega=\omega_{t}+\mathrm{B}_{f}+n
$$

Where:

$\omega \_$MEMS gyro measurement of angular velocity.

$\omega_{t} \_$MEMS gyro true angular velocity (unknown).

$\mathrm{B}_{f} \ldots$ MEMS gyro slow-changing component of the signal(gyroscope drift)

$n \_$MEMS gyro random noise (high frequency).

Since the temperature is the main factor, the precision of the gyroscope is impacted by ${ }^{\mathrm{B}_{f}}$ and $n$. The gyroscope drift is not only related to the measured temperature of the MEMS gyroscope but also is related to the temperature gradient of the surroundings. Additionally, the temperature gradient and the rate of temperature variation have a linear relationship; therefore, the drift of the MEMS gyroscope can be modeled as ${ }^{[3-4]}$ :

$$
\mathrm{B}_{f}=a * T+b * T^{\prime}+c
$$

Where:

$T$ _ The measured temperature of MEMS gyroscope.

$T^{\prime}$ _ Temperature variation rate of MEMS gyroscope.

$a, b, c \_$model parameters of MEMS gyroscope.

\section{Strong Adaptive Kalman Adaptive Filtering Compensation Algorithm}

In order to study and analyze the above model, solving the accurate model parameters is the main part of calculating the bias drift of MEMS gyroscope. The algorithm selects the parameter as the state variables, and the Strong Adaptive Kalman Adaptive Filter is established by the Equation (2). The model parameter of the MEMS gyroscope is estimated by the real-time updation of the strong tracking Kalman filter to ensure that it changes in a changing environment where there are also more accurate compensation effect.

$$
\begin{aligned}
& x_{k+1}=\Phi_{(k+1, k)} * x_{k}+\Gamma_{(k+1, k)} * \omega_{k} \\
& y_{k+1}=\mathrm{H}_{k+1} * x_{k+1}+v_{k+1}
\end{aligned}
$$


where:

$$
x_{k+1}=\left[\begin{array}{l}
a \\
b \\
c
\end{array}\right]_{k+1}, y_{k+1}=\left[\begin{array}{c}
m_{k+1} \\
m_{k} \\
m_{k-1}
\end{array}\right]
$$

$m_{k+1}$ —-Gyro bias measurement at time $\mathrm{k}+1 ; m_{k+1}=\hat{z}_{k}+\Delta A_{(k+1, k)} / \Delta t ; \hat{z}_{k}$ — The zero offset estimate calculated from the estimated model parameters at time $\mathrm{k}$;

$\Delta A_{(k+1, k)}$ — - The variation of the difference between the orientation of the compass and the orientation of the MEMS gyroscope from time $\mathrm{k}$ to time $\mathrm{k}+1$;

$$
\Delta A_{(k+1, k)}=\left(A_{-} \operatorname{compass}_{(k+1)}-A_{-} \text {Gyro }_{(k+1)}\right)-\left(A_{-} \operatorname{compass}_{(k)}-A_{-} G_{y r o}(k)\right) \text {; }
$$

where:

$A_{-} \operatorname{compass}_{(k+1)}, A_{-} \operatorname{Gyro}_{(k+1)}, A_{-} \operatorname{compass}_{(k)}, A_{-} \operatorname{Gyro}_{(k)}$ - - The orientations of the magnetic compass and MEMS gyro at time $\mathrm{k}$ to time $\mathrm{k}+1$;

$\Delta t$ _ — time interval from time $\mathrm{k}$ to time $\mathrm{k}+1$;

$\mathrm{H}_{\mathrm{k}+1}-$ measurement matrix of the the strong tracking Kalman filter ;

$$
\mathrm{H}_{k+1}=\left[\begin{array}{ccc}
T_{k+1} & T_{k+1}^{\prime} & 1 \\
T_{k} & T_{k}^{\prime} & 1 \\
T_{k-1} & T_{k-1}^{\prime} & 1
\end{array}\right]
$$

$T_{k+1}$ - Temperature measurement of MEMS gyro at time $\mathrm{k}+1$;

$T_{k+1}^{\prime}$ - - Temperature variation rate of MEMS gyro at time $\mathrm{k}+1$, it can be definited as:

$$
T_{k+1}^{\prime}=\left(T_{k+1}-T_{k}\right) / \Delta t
$$

In this paper, we reduce the bias drift by increasing the weight of the new measurements to avoid the error of the estimated value increase by the time.

The iterative equation of the new filter is as follows ${ }^{[5-6]}$ :

$$
\left.\begin{array}{l}
\left.\hat{x}_{k / k-1}^{*}=\Phi_{(k, k-1)} \hat{x}_{k-1}^{*}\right\} \\
\hat{x}_{k}^{*}=\hat{x}_{k / k-1}^{*}+K_{k}^{*}\left(y_{k}-\mathrm{H}_{k} \hat{x}_{k / k-1}^{*}\right) \\
K_{k}^{*}=P_{k / k-1}^{*} \mathrm{H}_{k}^{T}\left(\mathrm{H}_{k} P_{k / k-1}^{*} \mathrm{H}_{k}^{T}+R_{k}\right)^{-1} \\
P_{k / k-1}^{*}=\Phi_{(k, k-1)}\left(s P_{k-1}^{*}\right) \Phi_{(k, k-1)}^{T}+Q_{k-1} \\
P_{k}^{*}=\left(I-K_{k}^{*} \mathrm{H}_{k}\right) P_{k / k-1}^{*}
\end{array}\right\}
$$

It can be seen from equation (6) that the attenuation factor $s$ makes the new filter parameter $P_{k / k-1}^{*}$ greater than the parameters $P_{k / k-1}$ of the conventional filter, and it can get $K_{k}^{*}$ is larger than $K_{k}$, so when calculating $\hat{x}_{k}^{*}=\hat{x}_{k / k-1}^{*}+K_{k}^{*}\left(y_{k}-\mathrm{H}_{k} \hat{x}_{k / k-1}^{*}\right)=\left(1-K_{k}^{*} \mathrm{H}_{k}\right) \hat{x}_{k / k-1}^{*}+K_{k}^{*} y_{k}$, the weight of 
the previous measurements $\hat{x}_{k / k-1}^{*}$ decreases, and the weight of the current measurements $y_{k}$ increases. Considering the influence of frequency, the time interval for the strong tracking Kalman filter is set about every $10 \mathrm{~s}$. It can the parameters are not altered obviously at all the time and guarantee the accuracy of the compensation. In addition, the time interval can decrease the calculation.

\section{Analysis of experimental results}

In order to verify the error compensation effect of the improved algorithm, this experiment was carried out at a wide range of temperature $\left(23{ }^{\circ} \mathrm{C} \sim 57^{\circ} \mathrm{C}\right)$ in a static state of the system, analyzed the gyro bias under the drastic change of temperature in a short time, and tested the results.

In the whole experiment process, according to the information collected by the gyro can obtain system temperature and angular velocity measurement changes. Figure 1 shows that the temperature varied from $23^{\circ} \mathrm{C}$ to $57^{\circ} \mathrm{C}$ and then dropped down to room temperature. Figure 2 shows that the error of the gyroscope varied rapidly along with temperature. So the bias needs to be compensated accurately.

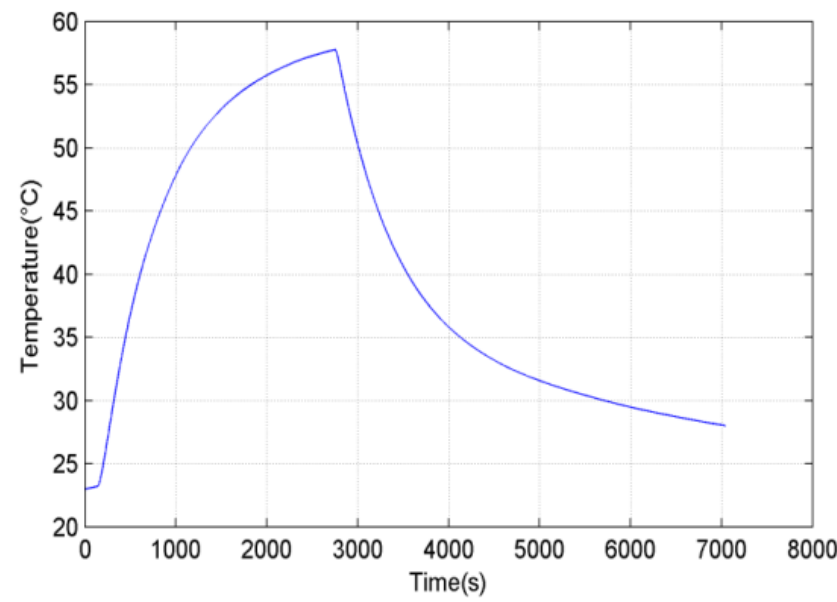

Figure 1 The variation of temperature when the gyroscope is static

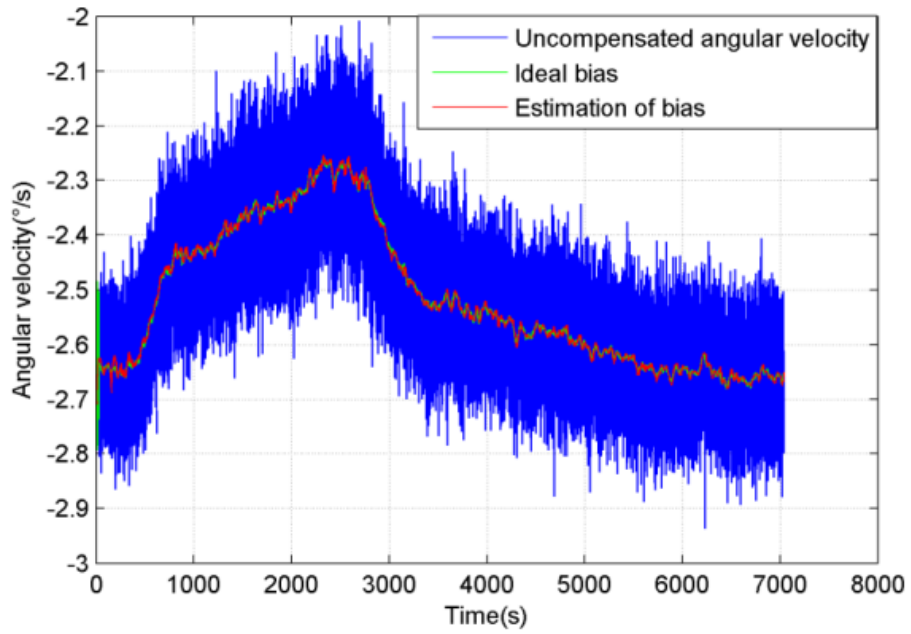

Figure 2 Uncompensated angular velocity, estimation bias and ideal bias of the gyroscope . 
At Figure 2, the blue line is the uncompensated angular velocity when the gyro is in a static temperature. The two figures show that the gyro angular velocity measurement changes with the outside temperature of the system. In addition, the gyro error will accumulate rapidly due to the inaccurate calculation of bias drift ${ }^{[7]}$. The red line indicates that the adaptive compensation method estimation of the gyroscope bias, the green line represents the ideal bias of the MEMS gyro.Compared with the two curves, it can be seen that the adaptive compensation method can estimate the deviation of the gyro accurately and ensure the compensation accuracy under the wide temperature range.

In order to verify the effect of the strong adaptive Kalman filter adaptive compensation algorithm, we did some experiments in the case of non-interference and interference. Moreover, in the entire process of the experiments, the heading error was made comparisons by using four algorithms. The results are shown in Figure 3,4.

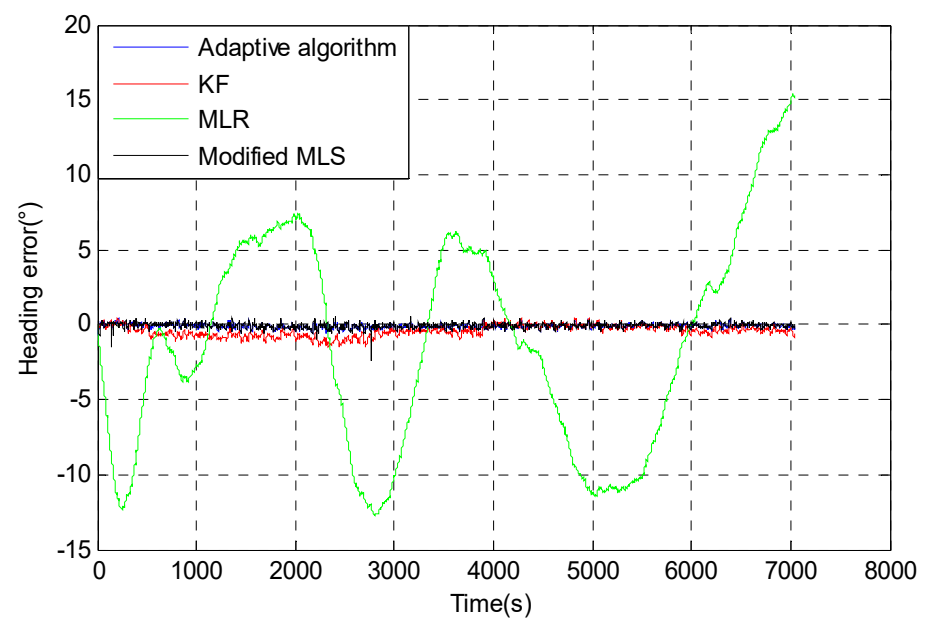

Figure 3 Heading error of the four algorithms without interference.

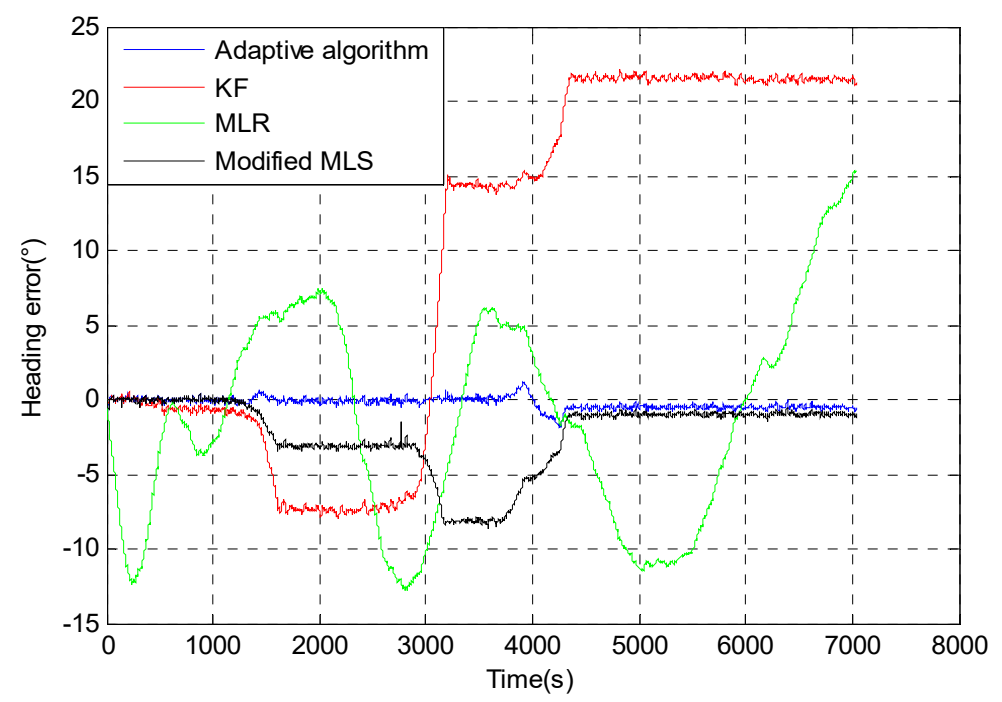

Figure 4 Heading error of the four algorithms with interference.

The blue line in the two figures shows the use of adaptive compensation algorithm, the red line shows the traditional Kalman filter compensation(KF), green line shows multiple linear regression compensation method(MLR), the black line shows the improved least squares method(LSM). To 
analyze the compensation performance of the temperature when the four algorithms do not work for some reason, we make the algorithms stop working by imposing magnetic field interference in three time periods $(300 \mathrm{~s}, 300 \mathrm{~s} \text { and } 600 \mathrm{~s})^{[8]}$. In the process of debugging, firstly fitting the initial value by the by least squares method, secondly the four algorithms are used to compensate the initial values respectively, finally the corresponding appropriate parameters and matrix equations are selected. In the process of calibrating, the adaptive algorithm is based on the observation variance to select the appropriate observation matrix and the parameters. Adjust the different parameters of the observation points to verify compensation performance of adaptive compensation algorithm, finally getting the appropriate adaptive observation parameters ${ }^{[9]}$. During the experiment, the noise of angular velocity is removed by wavelet domain denoising to reduce the bias. According to the static experiment, the compensation effects of the four algorithms are compared and analyzed under the condition of no interference and interference. The results are shown in table 1:

Table 1 Comparison and analysis of compensation effects of the four algorithms

\begin{tabular}{|c|c|c|c|c|c|}
\hline & & $\begin{array}{c}\text { Multiple linear } \\
\text { regression }\end{array}$ & $\begin{array}{c}\text { Traditional } \\
\text { Kalman filter }\end{array}$ & $\begin{array}{l}\text { Improved least } \\
\text { squares method }\end{array}$ & $\begin{array}{c}\text { Adaptive } \\
\text { compensation }\end{array}$ \\
\hline \multirow{2}{*}{$\begin{array}{l}\text { Zero bias of } \\
\text { static } \\
\text { experiment } \\
\text { without } \\
\text { interference }\end{array}$} & $\begin{array}{l}\text { mean } \\
\text { value }(\%)\end{array}$ & 0.002133 & $-1.1497 \times 10^{-4}$ & $-2.4517 \times 10^{-4}$ & $2.1620 \times 10^{-5}$ \\
\hline & $\begin{array}{l}\text { standard } \\
\text { deviation }\end{array}(\%)$ & 0.0235 & 0.0087 & 0.0107 & 0.0082 \\
\hline \multirow{2}{*}{$\begin{array}{l}\text { Zero bias of } \\
\text { static } \\
\text { experiment } \\
\text { with } \\
\text { interference }\end{array}$} & $\begin{array}{l}\text { mean } \\
\text { value }(\%) \text { s })\end{array}$ & 0.002133 & 0.003022 & $-2.0155 \times 10^{-4}$ & $-1.7512 \times 10^{-4}$ \\
\hline & $\begin{array}{l}\text { standard } \\
\text { deviation }\end{array}(\%)$ & 0.0235 & 0.0186 & 0.0136 & 0.0095 \\
\hline
\end{tabular}

According to Figure 3and Figure 4, the simulation results show that:

1.Adaptive compensation algorithm: 1) The error after compensation without interference is basically closed to 0 , and it ranged from $-0.5^{\circ}$ to $0.5^{\circ} ; 2$ ) The parameters can vary with the environment and have no effect when the environment is not drastically changed. The error ranged from $-0.6^{\circ}$ to $1.7^{\circ}$.

2.Traditional Kalman filter compensation: 1) The error without interference approximately ranged from $-1.6^{\circ}$ to $0.5^{\circ}$ in the whole process. Since updated parameters are constant, it is only applicable to the case where the temperature is slowly changing; 2 ) In the process of interference, the parameters of bias will stop updating and remain at the previous estimated error value. So the error is relatively large.

3.Multiple linear regression compensation method: In the process of interference and no interference the parameters remain constant all the time. Thus, the accuracy of compensation is constant over the whole temperature scope. The error ranged about from $-12.6^{\circ}$ to $15.1^{\circ}$.

4.Improved least squares compensation method: 1) It is almost the same as the adaptive compensation algorithm when there is no interference. The updated time interval of the model parameters is every 10 seconds, but the decrease of observation points of this method will cause the parameter error which will affect the compensation accuracy. 2) In the process of interference, the selection of sampling points caused errors in the parameters. The changes of temperature will have a greater impact on the bias and increase the heading error.

The table 1 shows that: In the static experiment without interference, the mean value of error after compensation with multiple linear regression method was about at $0.002 \%$; The traditional 
Kalman filter method improved the compensation precision to $-1.15 \times 10^{-4} \%$; The improved least squares method improved it to $-2.45 \times 10^{-4} \%$; The adaptive compensation method is the best, the mean value of error after compensation was about at $2.16 \times 10^{-5} \%$, and the standard deviation was less than the other three methods. In the static experiment with interference, the multiple linear regression method and the improved least squares method were not affected, and the compensation results of them were not changed; The influence of the traditional Kalman filtering method was relatively large. The compensation precision of adaptive compensation method is obviously better than the other three methods and the heading error compensated was much more accurate ${ }^{[10]}$.

In this paper, the adaptive Kalman filter compensation algorithm can maintain a high compensation precision when the temperature changes rapidly in a wide range, and the adaptive model parameters decrease the errors that the algorithm stops operating because of malfunctions in a short time.

\section{Conclusions}

In this paper, a new simplified iterative equation is established by using MEMS gyroscope combined with magnetic compass and accelerometer to calculate the corresponding zero bias model. Through a reasonable selection of the state quantity and the observation quantity of the equation, the bias drift is compensated accurately both in the process of interference and no interference. The results show that the strong tracking Kalman adaptive filtering algorithm can change parameters to adapt to the changes in the environment, which can decrease the error to the order of magnitude about $10^{-4} \%$. Also it can eliminate the influence of temperature and enhance the environmental adaptability of MEMS gyroscope. Thus, it can also effectively reduce the impact of the bias drift for a system which is integrated with MEMS gyroscopes and compasses.

\section{Acknowledgements}

This research was supported by National Natural Science Foundation of China (Project 61273082).

\section{References}

[1] N. Yazdi, F. Ayazi, K. Najafi, Micromachined inertial sensors,Proceedings of the IEEE 86 (8) (1998) 1640-1659.

[2]Qin Yongyuan, Zhang Hongyue, Wang Shuhua. Kalman filter and combined navigation principle [M]. Xi'an: Northwestern Polytechnical University Press, 1998.

[3] Jafari M, Najafabadi T A, Moshiri B, et al. PEM Stochastic Modeling for MEMS Inertial Sensors in Conventional and Redundant IMUs [J]. IEEE Sensors Journal, 2014.

[4] E. Sahin, S. E. Alper, T. Akin, Experimental evaluation of alternativedrive-mode control electronics developed for highperformanceMEMS gyroscopes, Transducers, Beijing, China, (2011)pp. 2817-2820.

[5] Zhao Xu, Su Zhong, Ma Xiaofei, et al. Study on zero - offset compensation of MEMS gyroscope under large temperature difference application environment [J]. Journal of Sensing Technology, 2012.

[6] Kim D, M'Closkey R T. Spectral Analysis of Vibratory Gyro Noise [J]. IEEE Sensors Journal, 2013.

[7] Kirkko-Jaakkola M, Collin J, Takala J. Bias prediction for MEMS gyroscopes [J]. IEEE Sensors Journal, 2012.

[8] Yang Xiaodong, Wang Wei. Geomagnetic navigation principle [M]. Beijing: National Defense Industry Press, (2009): 1-27.

[9] Xue L, Jiang C Y, Chang H L, et al. A novel Kalman filter for combining outputs of MEMS gyroscope array [J]. Measurement, 2012.

[10] Wang Chenxi. A Preliminary Study on the Digitalization of MEMS Gyro Peripheral Signal Processing Circuit (Master's Thesis) [D]. Jin Zhonghe, guidance. Hangzhou: Zhejiang University, 2006. 